\title{
Evidence for strategic suppression of irrelevant activation in the Simon task
}

\author{
Ronald Hübner*, Shreyasi Mishra \\ Universität Konstanz, Germany
}

\author{
PsycINFO classification: \\ 2323 \\ 2346 \\ Keywords: \\ Simon task \\ Activation suppression \\ Action control
}

\begin{abstract}
A B S T R A C T
It is well known that the Simon effect usually decreases with an increasing response time. According to a prominent account this is due to a gradually increasing suppression of irrelevant location induced activation. What is open, however, and what was investigated in the present study, is to what extent this selective sup pression can be adjusted strategically. We hypothesized that strategic suppression should depend on the availability of information about the inhibitory demands. Therefore, in two experiments the demand was modulated by varying the delay between a spatial cue and the target. In the first experiment, where target delay was randomized, there was a negative Simon effect for the longer delays. In a second experiment, where delay was blocked, the Simon effect remained positive. However, the overall Simon effect was larger than in Experiment 1. Together, our results show that the strength of selective suppression can be adjusted strategically, but that this does not necessarily lead to a smaller Simon effect.
\end{abstract}

\section{Introduction}

Dealing with opposing response tendencies is an important aspect of goal directed behavior, and several experimental conflict para digms, such as the Stroop task (Steinhauser \& Hübner, 2009; Stroop, 1935), or the Flanker task (Eriksen \& Eriksen, 1974; Hübner, Steinhauser, \& Lehle, 2010), have been developed for investigating the involved processes. In these paradigms internal stimulus features are commonly used to induce a response conflict. However, a conflict can also be induced by more external features such as location. This is obvious in the Simon task (Proctor, 2011; Simon, 1969), where a spa tial response (e.g. pressing a 'left' or 'right' button) to a target stimu lus is required, depending on the target's value on a non spatial dimension (e.g., its color or form). If the stimulus is then presented either to the left or right of fixation, responses are usually faster and more reliable when the target position is ipsilateral to the required response, compared to when it is contralateral. This Simon effect, which is in the focus of the present study, demonstrates that task irrelevant spatial information can have a substantial influence on response selection (for an overview see Hommel, 2011).

A widely acknowledged account of the Simon effect is the dual route idea (De Jong, Liang, \& Lauber, 1994; Kornblum, Hasbroucq, \& Osman, 1990), which assumes an indirect or controlled route, where task relevant information is intentionally translated into the required

\footnotetext{
* Corresponding author at: Universität Konstanz, Fachbereich Psychologie, Fach D29, D-78457 Konstanz, Germany. Tel.: + 497531882553.

E-mail address: Ronald.Huebner@uni-konstanz.de (R. Hübner).
}

response, and a direct route, along which the location of a stimulus au tomatically activates the spatially corresponding response code. The basic Simon effect is then explained by assuming that the automatic ac tivation of the response code via the direct route has a facilitating or in terfering influence on response selection, depending on the respective congruency of the trial.

A specific characteristic of the typical Simon effect is that it decreases with an increasing response time (RT), and that this holds for both la tencies and accuracy. That the congruency effect in accuracy decreases with RT is common to all conflict paradigms. It is large for short RTs, be cause of a high proportion of impulsive responses to the irrelevant in formation. The proportion then decreases with an increasing RT. In the latencies, the Simon effect usually also decreases with RT. This, how ever, is opposite to other conflict paradigms, such as the Flanker task, where the corresponding congruency effect in the latencies usually in creases with RT (e.g. Hübner et al., 2010). How the congruency effect changes with RT can be examined by considering so called delta func tions (e.g. De Jong et al., 1994; Ridderinkhof, 2002a), which directly specify the effect size as function of RT. The decreasing Simon effect in the latencies with RT is then reflected by a negative slope of the corre sponding delta function.

The relation between effect size and RT, as reflected by the delta functions, is very helpful for inferring the involved mechanisms (for an overview see van den Wildenberg et al., 2010). To explain the char acteristic time course of the Simon effect in the latencies, the temporal overlap hypothesis has been proposed. The idea is that the automatic activation induced by stimulus location decays with time (Hommel, 1993, 1994). Accordingly, on trials on which task relevant information 
is processed slowly, response translation takes place, at least partly, after the irrelevant location induced activation has decayed, so that the period of interference is relatively short. In contrast, on trials where the relevant information is processed rapidly, the translation process overlaps temporally to a large extent with the irrelevant activa tion, which produces a substantial interference.

Early support for the temporal overlap hypothesis was provided by Simon, Acosta, Mewaldt, and Speidel (1976). In their study they presented relevant as well as irrelevant information at the beginning of a trial, but the response had to be withheld until a go signal (tone) appeared at a variable delay. As expected, the Simon effect decreased with an increasing delay, and was absent at a delay of $250 \mathrm{~ms}$. Vallesi and Umiltà (2009) replicated this result, but also found that the Simon effect did not decrease completely when the relevant informa tion occurred after the irrelevant location information.

An important question with respect to the temporal overlap idea is whether the location induced activation decays passively, or whether some strategic suppression is necessary. Hommel (1994) investigated this question by manipulating not only the temporal overlap between irrelevant spatial activation and response selection, but also the proportion of congruent trials, which is known to affect the strategy of processing. Because no effect of proportion on the time course of the Simon effect was found, Hommel (1994) conclud ed that spatial activation decays passively and not due any strategic suppression.

However, in contrast to the passive decay hypothesis, Ridderinkhof (2002a, 2002b) proposed that irrelevant location induced activation is suppressed actively. In his activation suppression account he assumes that, to prevent unwanted responses to location, one needs to selective ly inhibit the response code that is automatically activated by the irrel evant location. Because it is assumed that suppression builds up gradually, though, it is more effective for relatively slow than for fast re sponses. Moreover, the strength of suppression depends on inhibitory demands. For instance, if the proportion of incongruent trials increases (e.g. Stürmer, Leuthold, Soetens, Schröter, \& Sommer, 2002), then selec tive suppression must be stronger. That suppression is indeed stronger under such conditions is reflected by a larger negative slope of the cor responding delta functions in the latencies (Ridderinkhof, 2002b).

Burle, van den Wildenberg, and Ridderinkhof (2005) tested the activation suppression account by laterally presenting an irrelevant cue before, simultaneously with, or after a bilateral target. They hy pothesized that activation followed by selective suppression should produce a negative effect when the location cue precedes the relevant information. Indeed, the Simon effect was positive for cues presented simultaneously with or after the target, but negative for cues appearing before the target. The fact that the Simon effect was nega tive if the spatial information was presented before the target strong ly supports the idea that irrelevant activation is suppressed. At the same time, however, it questions the assumption that the strength of suppression depends on the inhibitory demands, at least that the strength is adjusted appropriately. Obviously, suppression was too strong in Burle et al.'s (2005) experiment, otherwise there should have been no negative Simon effect.

The aim of the present study was to further investigate the details of activation suppression, especially, to what extent its strength can be ad justed strategically. The fact that the Simon effect was negative in Burle et al.'s (2005) study indicates that activation suppression was stronger than needed. However, this does not imply that the strength of activa tion suppression cannot be adjusted strategically. It should be noted that the passive decay hypothesis and the activation suppression hypothesis are not mutually exclusive. Therefore, it is possible that both processes are involved in the Simon task, and that suppression is used to accelerate the decay of activation. Thus, if one assumes that location induced activation always decays passively, then the inhibitory demand decreases with an increasing delay between the spatial cue and target. Accordingly, less suppression is needed for a long delay than for a short one. However, for an appropriate adjustment of suppression the inhibitory demand must be known in advance. This was not the case in Burle et al. (2005), because the delays were randomized. In contrast, in a similar experiment with blocked delays, Vallesi and Umiltà (2009) observed only positive Simon effects. This suggests that the participants in Burle et al.'s study applied more suppression than needed to prevent the Simon effect, at least for some delays.

In the present study these ideas were tested directly by conducting two experiments in which the delay between a spatial cue and the tar get was varied. Our procedure was similar to that of Experiment 2 in Vallesi and Umiltà's (2009) study, where first a peripheral spatial cue was presented that was then replaced by the target after a certain delay. However, instead of producing a further onset signal with the ap pearance of the target, we presented the target by deleting one cue component. As Vallesi \& Umiltá remarked, an onset target might pro duce an additional and delay independent activation of the spatial code, which reduces the delay effect.

More specifically, in our experiments we used left facing and right facing arrows $(<$, and $>)$ as stimuli. Spatial cues were constructed by superimposing both arrow types and by presenting them at one side of the display (see Fig. 1). After a certain delay one of the two arrows was removed, and the participants had to indicate the pointing direc tion of the remaining one. By this procedure task relevant informa tion could be delayed without producing a second onset signal that might produce an unwanted refresh of the location induced activa tion. Because with our procedure a delay of zero would have led to a qualitatively different location cue, we used only non zero delays. However, our shortest delay was $33 \mathrm{~ms}$, which might be considered as practically equivalent to a delay of zero.

In our first experiment, where the delays were randomized across trials, we tried to replicate the results of Burle et al. (2005) with our modified procedure. Then, for comparison, the delays were blocked in Experiment 2. If activation suppression is relatively unflexible, then the Simon effect should be similar in both experiments. Howev er, if participants can use the delay information in the blocked condi tion to strategically adapt their suppression strength accordingly, then performance should differ. In both experiment performance was examined by analyzing not only mean data, but also delta func tions for latencies and accuracy.

\section{Experiment 1}

In our first experiment several delays between a spatial cue and the target were randomized. First of all, we expected the Simon effect to decrease with an increasing delay. Moreover, if an appropriate ad justment of selective activation suppression should not be possible because of the randomized delays, then the Simon effect should be negative for the longer delays. The dynamics of activation suppres sion for the individual delays should be examined by also inspecting the corresponding delta functions.

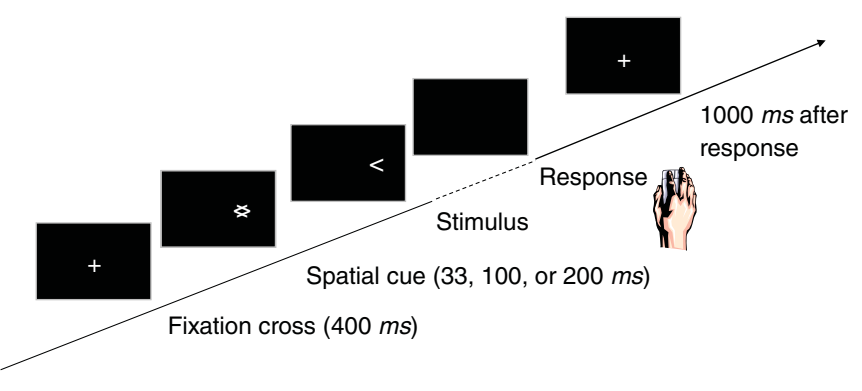

Fig. 1. Procedure of Experiment 1 


\subsection{Method}

\subsubsection{Participants}

17 students (mean age of 24 years; 5 male) from the Universität Konstanz, Germany, participated in the experiment. All had normal or corrected to normal vision and were paid $8 €$ for their participation.

\subsubsection{Stimuli, apparatus, and procedure}

Stimuli were right or left facing arrows $\left(<_{,}>\right)$. They were presented in white against a black background on an $18^{\prime \prime}$ color monitor with a res olution of $1280 \times 1024$ pixels, and a refresh rate of $60 \mathrm{~Hz}$. Participants were seated in front of the screen at a viewing distance of approximate ly $60 \mathrm{~cm}$. Each trial started with the presentation of a fixation cross at the center of the screen for $400 \mathrm{~ms}$, followed by a spatial cue consisting of two superimposed arrows, one facing to the left, the other facing to the right (see Fig. 1). The arrows appeared randomly at a distance of $2.2 \mathrm{~cm}$ left or right of the fixation cross, and were presented for 33 , 100 , or $200 \mathrm{~ms}$, after which the task irrelevant arrow was removed. These delays were chosen, because we expected the largest effects in this range (Simon et al., 1976). Delay was randomized across trials. The target arrow remained on screen until response. $1000 \mathrm{~ms}$ after the response the next trial started.

The task was to indicate the pointing direction of the target arrow by pressing one of the two mouse buttons (left button for left facing arrow; right button for right facing arrow) with the index or middle finger of the right hand, respectively. The participants were instructed to re spond as fast as possible without making many errors. On every incor rect trial, an auditory feedback was provided. At the end of each block the mean error rate and RT in that block was displayed. If the mean error rate exceeded $10 \%$ the participants were asked to be more accurate.

There was 1 practice block ( 30 trials), in which no spatial pre cue was presented, and 10 experimental blocks (96 trials/block). Every block contained an equal number of congruent and incongruent trials. On congruent trials, the cue (target) position was ipsilateral to the re quired response, while on incongruent trials it was contralateral.

\subsection{Results}

Responses faster than $100 \mathrm{~ms}$ or slower than $2000 \mathrm{~ms}$ were excluded from analysis $(<0.2 \%$ of all data).

The latencies of correct responses were analyzed by a two factor ANOVA for repeated measurements on the factors congruency (con gruent, or incongruent), and delay (33, 100, or $200 \mathrm{~ms}$ ). The analysis revealed a significant main effect of delay, $F(2,32)=18.9, p<.001$, $\eta_{\mathrm{p}}^{2}=.541$. RTs decreased with an increasing delay (412, 398, $391 \mathrm{~ms})$. However, there was also a significant interaction between delay and congruency, $F(2,32)=12.2, p<0.001, \eta_{\mathrm{p}}^{2}=.433$. The congruency effect was positive $(\Delta 14 \mathrm{~ms})$ for the $33 \mathrm{~ms}$ delay, but negative for the 100 and $200 \mathrm{~ms}$ delays $(\Delta-14 \mathrm{~ms}, \Delta-9 \mathrm{~ms})$. Congruency was significantly negative for the two longer delays, $F(1,16)=5.29, p<0.05, \eta_{\mathrm{p}}^{2}=.252$.

Mean error rate was $6.02 \%$. Subjecting the error rates to an ANOVA of the same type as for the latencies also revealed a significant main effect of delay, $F(2,32)=42.9, p<.001, \eta_{\mathrm{p}}^{2}=.728$. The error rate in creased with an increasing delay (1.67\%, 5.51\%, and 10.9\%). Congru ency did not produce a reliable effect nor a significant interaction with delay. Numerically, however, there was a positive Simon effect for the shortest delay $(\Delta 0.69)$, and a negative one for the longer de lays $(\Delta-2.10 \% ; \Delta-1.52 \%)$.

\subsubsection{Delta functions}

To compute the delta functions for the latencies (Fig. 2), we first computed the cumulative distribution functions for the RTs of correct responses for each congruency condition (congruent, incongruent), and delay $(33,100,200 \mathrm{~ms})$ by quantile averaging $(.1, .3, .5, .7, .9)$

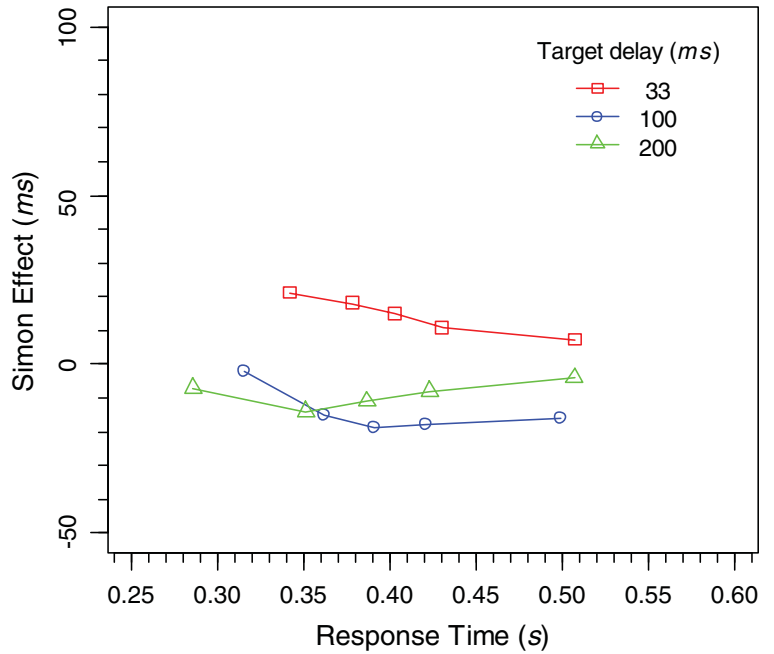

Fig. 2. Delta functions in the latencies of correct responses in the different target-delay conditions in Experiment 1.

the data (Ratcliff, 1979). Delta functions were then obtained by calcu lating the differences of the corresponding quantiles between the congruent and incongruent conditions and relating them to their respective average RTs (see Fig. 2). For a statistical analysis, the quantiles for the individual delay conditions and delays were entered into thee factor ANOVAs for repeated measurements on the factors congruency (congruent, or incongruent), delay (33, 100, or $200 \mathrm{~ms}$ ), and quantile (1 to 5 ). We only report relevant results involving the factor quantile.

The analysis revealed a significant three way interaction between all factors, $F(8,128)=2.69, p<0.01, \eta_{\mathrm{p}}^{2}=.144$, indicating that the variation of the Simon effect with RT differed between the delay con ditions. Further analyses showed that the Simon effect in the latencies changed significantly with RT for delay $33 \mathrm{~ms}, F(4,64)=2.82$, $p<0.05, \eta_{\mathrm{p}}^{2}=.150$, and for delay $100 \mathrm{~ms}, F(4,64)=4.07, p<0.01$, $\eta_{\mathrm{p}}^{2}=.203$, but not for delay $200 \mathrm{~ms}$ (see Fig. 2). Moreover, the varia tion did not differ reliably between the two shortest delays.

To obtain delta functions for accuracy, we first calculated condi tional accuracy functions for each participant and condition. For this objective the corresponding data were sorted into five $20 \%$ bins. Then the proportion of correct responses and the mean RT for each bin were computed and the resulting values were averaged across participants. Delta functions were then constructed by calculating

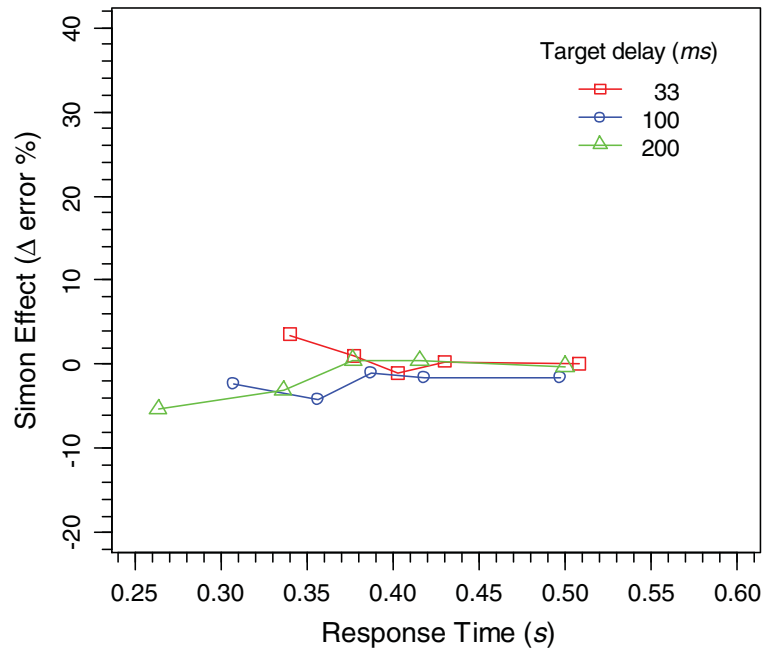

Fig. 3. Delta functions for accuracy in the different target-delay conditions in Experiment 1. 
the differences in accuracy of the corresponding bins between congruent and incongruent conditions, and relating them to their averaged RT (see Fig. 3). Statistical analyses were performed analo gously to those for the RT delta functions.

As can be seen in Fig. 3, the delta functions in accuracy were rather flat. Accordingly, there was no significant variation of the Simon effect with RT.

\subsection{Discussion}

The results of this experiment show that the delays had a substan tial impact on the Simon effect. It was positive for the shortest delay, but negative for the two longer delays. Thus, our results are similar to those of Burle et al. (2005). The fact that the Simon effect was nega tive for the longer delays indicates that there was selective activation suppression. At the same time, however, the negative effects suggest that the strength of suppression was not optimal for each of the indi vidual delays. Rather it seems that the participants chose a suppres sion strength that was presumably appropriate for the shortest delay, but too strong for the longer ones.

That selective activation suppression was rather strong is also reflected by the delta functions. If we consider the functions for the latencies (Fig. 2), then we see that for the shortest delay the Simon ef fect decreased monotonically with RT and almost reached zero for the slowest responses. For the $100 \mathrm{~ms}$ delay, however, overall suppres sion strength was so strong that the Simon effect was already absent for the fastest responses, and then became increasingly negative until it leveled off.

In the mean error rates no reliable Simon effect occurred, although numerically the pattern was similar to that in the mean RTs. If we consider the delta functions for accuracy (Fig. 3), then we see that the Simon effect was absent not only for the slow responses, but also for the faster ones. The fact that the Simon effect was practically absent even for the fastest responses indicates that impulsive re sponses were largely prevented right from the beginning of process ing. Because selective suppression is assumed to affect mainly slow responses, this prevention must have been due to some other process. It is conceivable, for instance, that the susceptibility for spatial infor mation was generally reduced (van den Wildenberg et al., 2010).

Taken together, the present results indicate that there was selec tive suppression, but that its strength was not adapted to the inhibi tory demands of the individual delay conditions. However, this does not imply that suppression can principally not be adapted strategical ly. Rather, it could be that adaptation was not possible in the present case, because the delay for a given trial was not known in advance. Consequently, participants chose a strength that met the average de mands across delays. Whether the strength of suppression can be controlled strategically, if the inhibitory demand is known in advance on each trial, was tested in the next experiment.

\section{Experiment 2}

This experiment was similar to our first one, except that delay was blocked. If the results in Experiment 1 were indeed due to a general inability to adapt the strength of selective suppression to the trial based inhibitory demands, then the same results as in Experiment 1 should be observed. However, if the strength can be adjusted strate gically, given the demands are known in advance on each trial, then blocking the delays should allow an appropriate adjustment. Accord ingly, it can be expected that an individual and optimal suppression strength will be applied for each delay. If this is the case, and if we further assume that location induced activation cannot fully be suppressed for the shortest delay, then the Simon effect should also decrease with an increasing delay. However, it should approach zero, and not be negative, even for the longest delay.

\subsection{Method}

17 students (mean age of 24 years; 3 male) from the Universität Konstanz, Germany, participated in the experiment. All had normal or corrected to normal vision and were paid $8 €$ for their participation. Apparatus and stimuli were same as in Experiment 1. The procedure was also similar, except that the delays were blocked. Additional to the practice block there were 5 experimental blocks ( 64 trials/block) for each of the three delays. The order of delays was balanced across participants.

\subsection{Results}

Responses faster than $100 \mathrm{~ms}$ or slower than $2000 \mathrm{~ms}$ were excluded from data analysis ( $<0.3 \%$ of all data).

The latencies of correct responses were subjected to a two factor ANOVA for repeated measurements on the factors congruency (con gruent, or incongruent), and delay (33, 100, or $200 \mathrm{~ms})$. The analysis revealed significant main effects of both congruency, $F(1,16)=13.7$, $p<.01, \eta_{\mathrm{p}}^{2}=.462$, and delay, $F(2,32)=6.19, p<.01, \eta_{\mathrm{p}}^{2}=.279$. Re sponses were faster for congruent compared to incongruent stimuli ( $407 \mathrm{~ms}$ versus $423 \mathrm{~ms}$ ), and speed increased with an increasing delay $(422,417,407 \mathrm{~ms})$. A further test revealed that the mean RT was also significantly different between the two longest delays, $F(1$, $16)=8.44, p<.05, \eta_{\mathrm{p}}^{2}=.345$. However, there was a significant in teraction between congruency and delay, $F(2,32)=18.9, p<.001$, $\eta_{\mathrm{p}}^{2}=.541$, indicating that the congruency effect varied with delay $(\Delta 34, \Delta 3, \Delta 11 \mathrm{~ms})$. A further analysis revealed that the Simon effect was no longer significant when the shortest delay was excluded, $F(1,16)=2.00, p=0.177, \eta_{\mathrm{p}}^{2}=.088$.

Mean error rate was 6.75\%. Subjecting the error rates to an ANOVA of the same type as for the response times also revealed a significant main effect of congruency, $F(1,16)=22.0, p<0.001, \eta_{\mathrm{p}}^{2}=.579$, and of delay, $F(2,32)=3.60, p<.05, \eta_{\mathrm{p}}^{2}=.184$. The error rate was smaller for con gruent than for incongruent stimuli (4.97\% versus $8.53 \%$ ), and increased with delay (5.85\%, 6.71\%, 7.69\%). However, congruency interacted signif icantly with delay, $F(2,32)=3.38, p<.05, \eta_{\mathrm{p}}^{2}=.175$, indicating that the congruency effect was larger for the $33 \mathrm{~ms}$ delay than for the other delays $(\Delta 5.82 \%, \Delta 2.18 \%, \Delta 2.70 \%)$. However, a further analysis revealed that the Simon effect remains significant after excluding the shortest delay, $F(1,16)=4.58, p<0.05, \eta_{p}^{2}=.223$.

\subsubsection{Delta functions}

Delta functions were computed and analyzed in the same way as in Experiment 1. The analysis revealed a significant three way inter action between all factors, $F(8,128)=3.73, p<0.001, \eta_{\mathrm{p}}^{2}=.189$, which indicates that the variation of the Simon effect with RT differed between the delay conditions. Further analyses showed that the var iation was significant for delay $100 \mathrm{~ms}, F(4,64)=4.40, p<0.01$, $\eta_{\mathrm{p}}^{2}=.216$, and marginally significant for delay $33 \mathrm{~ms}, F(4,64)=$ $2.27, p=0.071, \eta_{\mathrm{p}}^{2}=.124$. The Simon effect did not vary reliably for delay $200 \mathrm{~ms}$.

Fig. 5 shows the delta functions for accuracy. As can be seen, the Simon effect was relatively large for the fastest responses, especially for the shortest delay. It then decreased quickly with RT and approached zero. The significant three way interaction between all factors, $F(8$, $128)=3.68, p<0.001, \eta_{p}^{2}=.187$, indicates that the decrease of the Simon effect in accuracy with RT differed between the delay conditions. Further analyses revealed that it was significant for delay $33 \mathrm{~ms}, F(4$, $64)=23.4, p<0.001, \eta_{\mathrm{p}}^{2}=.594$, and for delay $100 \mathrm{~ms}, F(4,64)=$ $5.96, p<0.01, \eta_{\mathrm{p}}^{2}=.271$, but not for delay $200 \mathrm{~ms}$.

\subsubsection{Comparison with Experiment 1}

For comparison, the mean data of the present experiment were combined with those of Experiment 1 and subjected to three factor ANOVAs for the within participant factors congruency (congruent, or 
incongruent), and delay (33,100, or $200 \mathrm{~ms}$ ), and the between par ticipant factor experiment (randomized delay, or blocked delay). We report only results involving the factor experiment.

For the latencies the analysis revealed a significant interaction be tween experiment and congruency, $F(1,32)=11.9, p<.01, \eta_{\mathrm{p}}^{2}=.270$. The Simon effect was smaller for randomized delays than for blocked ones. This is even the case when we compare the absolute effect sizes, i.e. ignore their sign, $F(1,32)=5.75, p<.05, \eta_{\mathrm{p}}^{2}=.152$. Moreover the interaction between all three factors was far from significance, $F(2,64)=.107, p=.899, \eta_{\mathrm{p}}^{2}=.003$. This implies that the Simon effect for the shortest delay was also smaller under randomized delays, com pared to blocked ones, which we nevertheless confirmed separately, $F(1,32)=16.5, p<.001, \eta_{\mathrm{p}}^{2}=.340$.

For the error rates the analysis also revealed a significant interaction between experiment and congruency, $F(1,32)=15.7, p<.001, \eta_{\mathrm{p}}^{2}=$ .330. The overall Simon effect was small and negative for randomized delays, but large and positive for blocked ones. Moreover, there was an interaction between experiment and delay, $F(2,64)=18.8$ $p<.001, \eta_{\mathrm{p}}^{2}=.371$. This was due to the fact that the error rate in creased only slightly with delay in the blocked delay condition, but sub stantially in the randomized condition.

We also examined the differences of the delta functions between the two experiments by comparing the corresponding functions for each delay. In the latencies, merely the functions for delay $100 \mathrm{~ms}$ dif fered significantly, $F(4,128)=3.23, p<.05, \eta_{\mathrm{p}}^{2}=.092$. In accuracy, the functions for delay $33 \mathrm{~ms}$ differed reliably, $F(4,128)=16.0$, $p<.001, \eta_{\mathrm{p}}^{2}=.333$, as did the functions for delay $100 \mathrm{~ms}, F(4$, $128)=3.18, p<.05, \eta_{\mathrm{p}}^{2}=.091$.

\subsection{Discussion}

Our results show that the Simon effect also varied with blocked delays. However, different from Experiment 1, the effect remained positive. There was a substantial Simon effect for the shortest delay, whereas for the longer delays it was reliable only in the error rates. Most importantly, it remained positive. This is in contrast to Burle et al.'s (2005) results and our Experiment 1, where strong negative Simon effects were found. Because delay was blocked in the present experiment, the results support our idea that the strength of suppres sion can be adjusted according to the inhibitory demands for the indi vidual delay conditions, but only if the delay is known in advance.

Different from our expectation, though, the Simon effect did not monotonically decrease with an increasing delay. Rather, at least nu merically, the effect was stronger for the 200 ms delay than for the 100 ms delay. However, because mean RT was reliably shorter for the 200 ms delay, compared to the $100 \mathrm{~ms}$ delay, this tendency could have been due to some kind of tradeoff.

The variation of the Simon effects with RT for the individual delays can be seen by inspecting the corresponding delta functions (Figs. 4 and 5). The functions for the latencies show that the Simon effect for the shortest delay first increased with RT and then decreased. However, it remained relatively strong across the whole RT range. These data are similar to those of Baroni, Pellicano, Lugli, Nicoletti, and Proctor (2012), who also presented lateralized arrows as targets, but without a prior spatial cue. The similarity between these data sets confirms our assumption that our shortest delay is practically equiv alent to a delay of zero. For the longer delays the Simon effect was smaller, compared to the short delay, and the corresponding delta functions were concave rather than convex. That is, the effect first de creased to a minimum and then increased. However, this variation was reliably only for delay $100 \mathrm{~ms}$.

If we consider the delta functions for accuracy (Fig. 5), then we see that they are rather different from those in the previous experiment. The Simon effect was rather large for the fastest responses, especially for the shortest delay. However, the effect quickly vanished with RT for all conditions. The large Simon effect in accuracy for fast responses

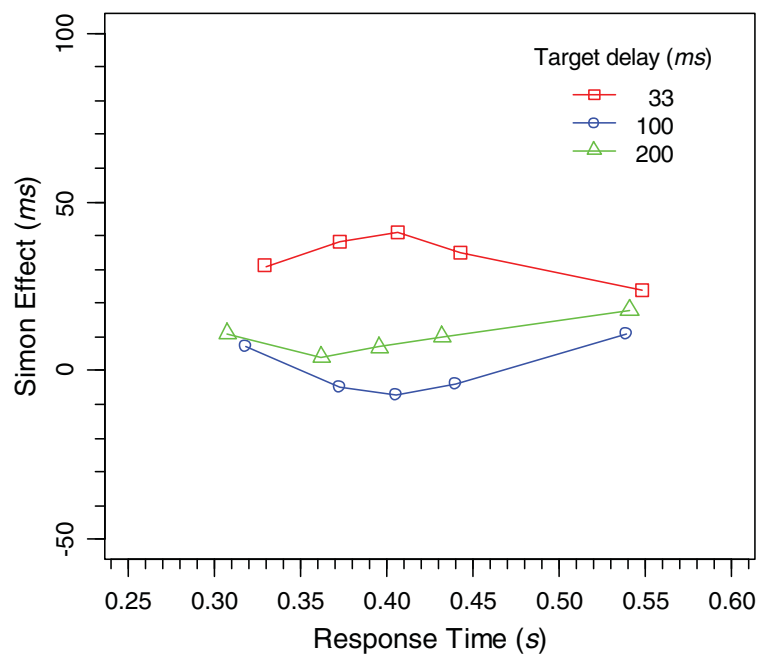

Fig. 4. Delta functions for latencies of correct responses in the different target-delay conditions in Experiment 2.

indicates that the readiness for responding impulsively to irrelevant spatial information was relatively large, compared to our first exper iment with randomized delays.

\section{General discussion}

The aim of the present study was to further investigate selective activation suppression (Ridderinkhof, 2002a, 2002b) in the Simon task, especially to what extent its strength can strategically be adjust ed to inhibitory demands. We assumed that location induced activa tion automatically decays with time and that, therefore, the inhibitory demand decreases with an increasing temporal separation of irrele vant activation and response selection (Hommel, 1993, 1994). Thus, one way to modulate the inhibitory demand is to vary the delay between an irrelevant spatial cue and the target. By applying such a procedure, Burle et al. (2005) found a strong negative Simon effect when the cue preceded the target, which speaks for selective sup pression, but questions that it can strategically be adjusted optimally. However, in that study the delays were randomized so that no infor mation about the inhibitory demand on a given trial was available in advance. In a similar experiment but with blocked delays Vallesi and Umiltà (2009) found that the Simon effect remained positive. Thus,

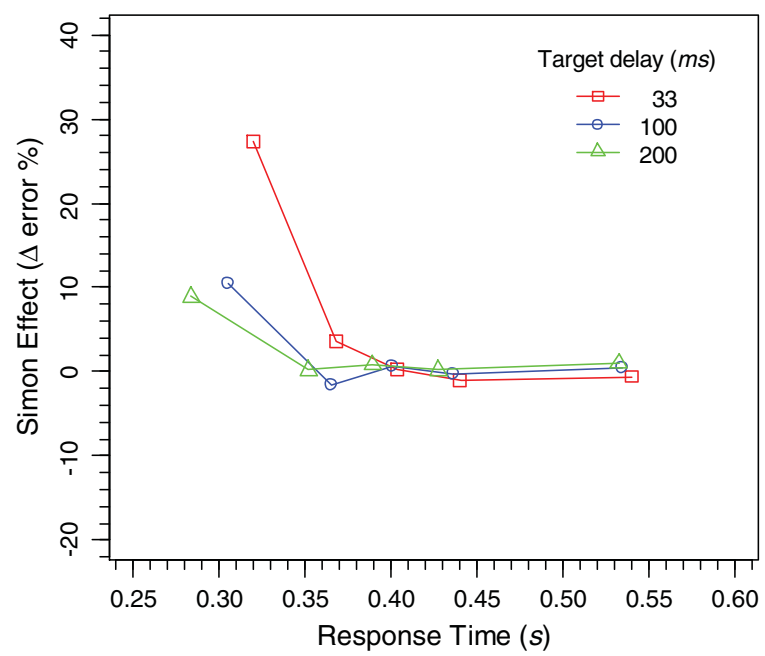

Fig. 5. Delta functions for accuracy in the different target-delay conditions in Experiment 2 . 
our idea was to examine whether strategic suppression is possible if such information is provided.

In our first experiment, three delays were randomized in a block of trials to see whether Burle et al.'s (2005) results can be replicated with a slightly modified procedure (e.g., offset stimuli and different delays). As a result, the Simon effect was positive only for the shortest delay, but negative for the two longer delays, confirming Burle et al.'s outcome. Thus, our data also indicated that location induced activa tion was not suppressed in dependence of the inhibitory demands of the individual delays. Rather, it seemed that the same strength of suppression was applied for all delays. The strength was largely appropriate for the shortest delay, but for the longer delays, which re quired less inhibition, it was too high.

If location induced activation can be suppressed strategically, but only if the inhibitory demands are known in advance, then the Simon effect should vary differently with delay when the delays are blocked. Whether this is the case was tested in Experiment 2. The results show that the Simon effect was again large and positive for the shortest delay. However, and most importantly, although the effect also de creased for the longer delays, it remained positive. This supports the idea of strategic activation suppression. The result can be interpreted in the sense that selective suppression was relatively strong for the shortest delay. Nevertheless, because suppression takes some time to come into effect, and is probably also limited in strength, it could not prevent that irrelevant activation strongly affected response selection. For the longer delays, the demands were lower, because location based activation had partly decayed and suppression had more time to act. Since the participants knew the demands in advance, they could adjust their suppression strength accordingly, so that the Simon effect was absent in RT and relatively weak in the error rates.

How selective suppression proceeded in the different experiments and conditions can, at least partly, be inferred from the delta func tions. If we consider Fig. 4, then, taken together, the functions for the different delays suggest that irrelevant spatial activation in creased after cue onset and was then suppressed for some time. Inter estingly, with randomized delays the dynamics of suppression was rather similar (see Fig. 2). It merely seems that suppression started somewhat earlier and continued for a longer period, which suggests that participants had some control of the timing of suppression. The most striking difference of the delta functions for the latencies, how ever, was that they were shift downwards for randomized delays (Experiment 1). This could indicate that suppression was generally stronger in this case. However, given that selective suppression takes some time to take effect, as assumed by the activation suppression model, one would have expected a larger difference in shape of the delta functions between the experiments, especially for slow responses. Yet, only one (delay $100 \mathrm{~ms}$ ) of the three delta functions differed significant ly between the experiments.

Thus, it seems that some other mechanism was also involved in producing the differences in performance between the experiments. It has been suggested that the readiness for making fast impulsive re sponses to irrelevant information is also under voluntary control, at least to some extent, and that this readiness is primarily reflected by the shape of the delta functions for accuracy (e.g. van den Wildenberg et al., 2010). The more impulsive responses to location are allowed, the larger the congruency effect in the error rates for fast responses. That the readiness for impulsive responses indeed dif fered between our experiments can be seen by comparing the corre sponding delta functions for accuracy. For randomized delays there were only few impulsive responses to location (see Fig. 3), i.e. the Simon effect was practically absent even for the fastest responses, whereas for blocked delays many such fast responses occurred, which produced a correspondingly large Simon effect (Fig. 5), espe cially for the shortest delay.

Alternatively, it is conceivable that under randomized delays the flow of information along the direct route was generally blocked.
Such a mechanism would not only account for the different patterns of the delta functions for accuracy between experiments, but also for the vertical shift of the delta functions for the latencies, i.e. for the fact that the Simon effect was generally smaller under random ized delays. In any case, the analyses of the delta functions strongly suggest that two mechanisms were applied by the participants to adapt to the different demands in our experiments.

Thus, taken together, our results show that suppression strength can be adjusted according to the demands, given they are known in advance. Moreover, they suggest that also the readiness to respond impulsively to location is under strategic control, and that this readi ness is reduced if there is uncertainty about target arrival. However, our results also indicate that the different adjustment do not neces sarily produce the smallest possible Simon effects. The comparison of the Simon effect between the two experiments revealed that it was reliably larger under blocked delays than under randomized ones. This was even the case when we consider the absolute effect sizes (i.e. ignore the sign). Thus, the possibility to adjust suppression to the individual delays did not lead to a smaller overall Simon effect, as one might have expected, but to a larger one.

How can this unexpected result be explained? Implicitly, one might have assumed that the participants' goal was to prevent the Simon effect. This, however, was obviously not the case. Rather, our data suggest that the participants' goal was to meet our requirements concerning accuracy. This makes sense, because, first of all, they pre sumably did not notice the size of the Simon effect. Furthermore, what they certainly noticed, also due to our feedback procedure, was their error rate. Accordingly, the overall error rate was rather similar between the two experiments (6\% versus $7 \%$ ). For the blocked delays (Experiment 2 ), a similar error rate also occurred for the indi vidual delays $(6 \%, 7 \%, 8 \%)$. For the randomized delays (Experiment 1 ), however, the individual error rates differed substantially, i.e. they in creased considerably with delay $(2 \%, 6 \%, 11 \%)$.

These results indicate that location induced activation had to be uti lized to a lesser extent and suppressed more strongly in the random condition to perform as reliably, on average, as in the blocked condition, which also resulted in a smaller Simon effect. This interpretation also explains why the Simon effect for the shortest delay was larger in the blocked condition than in the randomized one. Although it was obvi ously possible in the blocked condition to suppress location induced ac tivation more strongly, it was not necessary, because the required accuracy was already achieved with less suppression. Moreover, it should be noticed that activation suppression also produces costs on congruent trials. Thus, it should not always be maximized. These con siderations suggest that selective activation suppression was not used in our experiments to minimize the Simon effect, but to control the level of accuracy. Nevertheless, our results support the idea that activa tion suppression can be adjusted strategically.

To conclude, the present study demonstrates that the effect of irrel evant location information in the Simon task can strategically be con trolled, depending on the situational demands. Our results suggest that two mechanisms are applied. One mechanism, selective activation suppression, develops in time and is used to reduce the irrelevant acti vation. This mechanism can effectively be adapted to the specific de mands of individual delays, given they are known in advance. The other mechanism controls the utilization of location information. The use of location information for response selection is reduced if the adap tation of the first mechanism to individual conditions is largely restrict ed, which is the case with randomized delays. In any case, the primary goal of these strategic modulations is not to minimize the Simon effect, but to meet the situational requirements with respect to accuracy.

\section{Acknowledgments}

We thank Lisa Töbel and two anonymous reviewers for their valuable comments concerning this paper. 


\section{References}

Baroni, G., Pellicano, A., Lugli, L., Nicoletti, R., \& Proctor, R. W. (2012). Influence of temporal overlap on time course of the Simon effect. Experimental Psychology, 59, $88-98$

Burle, B., Wildenberg van den, W. P. M., \& Ridderinkhof, K. R. (2005). Dynamics of facilitation and interference in cue-priming and Simon tasks. European Journal of $\mathrm{Cog}_{-}$ nitive Psychology, 17, 619-641.

De Jong, R., Liang, C. C., \& Lauber, E. (1994). Conditional and unconditional automaticity: A dual-process model of effects of spatial stimulus-response correspondence. Journal of Experimental Psychology. Human Perception and Performance, 20 731-750.

Eriksen, B. A., \& Eriksen, C. W. (1974). Effects of noise letters upon the identification of a target letter in a nonsearch task. Perception \& Psychophysics, 16, 143-149.

Hommel, B. (1993). The relationship between stimulus processing and response selec tion in the Simon task: Evidence for a temporal overlap. Psychological Research, 55 , 280-290.

Hommel, B. (1994). Spontaneous decay of response-code activation. Psychological Research, 56, 261-268.

Hommel, B. (2011). The Simon effect as tool and heuristic. Acta Psychologica, 136 189-202.

Hübner, R., Steinhauser, M., \& Lehle, C. (2010). A dual-stage two-phase model of selective attention. Psychological Review, 117, 759-784.

Kornblum, S., Hasbroucq, T., \& Osman, A. (1990). Dimensional overlap: Cognitive basis for stimulus-response compatibility - A model and taxonomy. Psychological Review, 97, 253-270.

Proctor, R. W. (2011). Playing the Simon game: Use of the Simon task for investigating human information processing. Acta Psychologica, 136, 182-188.
Ratcliff, R (1979). Group reaction time distributions and the analysis of distribution statistics. Psychological Bulletin, 86, 446-461.

Ridderinkhof, K. R. (2002b). Activation and suppression in conflict tasks: Empirical clarification through distributional analyses. In W. Prinz, \& B. Hommel (Eds.) Common mechanisms in perception and action. Attention \& performance, vol. XIX. (pp. 494-519). Oxford: Oxford University Press.

Ridderinkhof, K. R. (2002a). Micro- and macro-adjustments of task set: Activation and suppression in conflict tasks. Psychological Research, 66, 312-323.

Simon, J. R. (1969). Reactions toward the source of stimulation. Journal of Experimental Psychology, 81, 174-176.

Simon, J. R., Acosta, E., Mewaldt, S. P., \& Speidel, C. R. (1976). The effect of an irrelevant directional cue on choice reaction time: Duration of the phenomenon and its relation to stages of processing. Perception \& Psychophysics, 19, 16-22.

Steinhauser, M., \& Hübner, R. (2009). Distinguishing response conflict and task conflict in the Stroop task: Evidence from ex-Gaussian distribution analysis. Journal of Experimental Psychology. Human Perception and Performance, 35, 1398-1412.

Stroop, J. R. (1935). Studies of interference in serial verbal reactions. Journal of Experimental Psychology, 18, 643-662.

Stürmer, B., Leuthold, H., Soetens, E., Schröter, H., \& Sommer, W. (2002). Control over location-based response activation in the Simon task: Behavioral and electrophysiological evidence. Journal of Experimental Psychology. Human Perception and Performance, $28,1345-1363$

Vallesi, A., \& Umiltà, C. A. (2009). Decay of stimulus spatial code in horizontal and vertical Simon tasks. The Journal of General Psychology, 136, 350-373.

van den Wildenberg, W. P. M., Wylie, S. A., Forstmann, B. U., Burle, B., Hasbroucq, T., \& Ridderinkhof, K. R. (2010). To head or to heed? Beyond the surface of selective action inhibition: A review. Frontiers in Human Neuroscience, 4, 222 